\title{
Regulation of cancer cell migration and bone metastasis by RANKL
}

\author{
D. Holstead Jones ${ }^{1,2,3 \star} \uparrow$, Tomoki Nakashima ${ }^{1 \star}$, Otto H. Sanchez ${ }^{4} \dagger$, Ivona Kozieradzki ${ }^{1,2,3}$, Svetlana V. Komarova ${ }^{5}$, \\ Ildiko Sarosi ${ }^{6}$, Sean Morony ${ }^{6}$, Evelyn Rubin ${ }^{2,3}$, Renu Sarao ${ }^{1}$, Carlo V. Hojilla ${ }^{4}$, Vukoslav Komnenovic ${ }^{1}$, \\ Young-Yun Kong ${ }^{7}$, Martin Schreiber ${ }^{8}$, S. Jeffrey Dixon ${ }^{9}$, Stephen M. Sims ${ }^{9}$, Rama Khokha ${ }^{2,4}$, Teiji Wada \\ $\&$ Josef M. Penninger ${ }^{1,2,3}$
}

Bone metastases are a frequent complication of many cancers that result in severe disease burden and pain ${ }^{1-3}$. Since the late nineteenth century, it has been thought that the microenvironment of the local host tissue actively participates in the propensity of certain cancers to metastasize to specific organs, and that bone provides an especially fertile 'soil'4 . In the case of breast cancers, the local chemokine milieu is now emerging as an explanation for why these tumours preferentially metastasize to certain organs ${ }^{5}$. However, as the inhibition of chemokine receptors in vivo only partially blocks metastatic behaviour ${ }^{6}$, other factors must exist that regulate the preferential metastasis of breast cancer cells. Here we show that the cytokine RANKL (receptor activator of NF- $\mathrm{kB}$ ligand $)^{7,8}$ triggers migration of human epithelial cancer cells and melanoma cells that express the receptor RANK. RANK is expressed on cancer cell lines and breast cancer cells in patients. In a mouse model of melanoma metastasis ${ }^{9}$, in vivo neutralization of RANKL by osteoprotegerin results in complete protection from paralysis and a marked reduction in tumour burden in bones but not in other organs. Our data show that local differentiation factors such as RANKL have an important role in cell migration and the tissue-specific metastatic behaviour of cancer cells.

RANKL (also referred to as OPGL, TRANCE or ODF) is a member of the tumour necrosis factor (TNF) family of cytokines that binds to its receptor RANK to control osteoclast differentiation, activation and survival ${ }^{7,8,10}$. Osteoprotegerin $(\mathrm{OPG})$ is a soluble decoy receptor for RANKL that blocks ligand binding to RANK, thereby preventing the signalling required for osteoclast differentiation and activation ${ }^{11}$. RANK is also constitutively expressed in normal mammary gland epithelial cells, but RANKL expression is induced by sex hormones during pregnancy ${ }^{12}$. Genetically, both RANKL and RANK are essential for the development of the lactating mammary gland during pregnancy ${ }^{12}$ and for lymph node organogenesis in mouse embryos ${ }^{7}$.

This unexpected distribution of RANKL led us to examine multiple epithelial tissues for RANK expression. In all mouse epithelial tissues analysed, RANK messenger RNA was present, even in epithelial tissues of the early embryo. We also detected marked RANK expression in a large number of primary human breast tumour samples as well as in cancer cells present in local lymph node metastases (Fig. 1a and Supplementary Fig. 1a). Moreover, several human prostate and breast cancer cell lines, but not colon cancer cell lines, expressed RANK mRNA (Supplementary Fig. 1b), and RANK protein was detected on the surface of breast cancer cells by fluorescein isothiocyanate (FITC)-labelled RANKL binding (Fig. 1b and Supplementary Fig. 2a). Stimulation of RANK-positive human breast cancer cells with recombinant RANKL induced strong actin polymerization that could be blocked by OPG (Fig. 1c). Although RANK stimulation resulted in enhanced activation of protein kinase $\mathrm{B}(\mathrm{PKB} / \mathrm{AKT})$ and extracellular signal-regulated kinases 1 and 2 (ERK1/2) (Supplementary Fig. 2b, c), RANKL had no apparent effect on proliferation or death susceptibility of these epithelial tumour cells (Supplementary Fig. 2d, e). Thus, RANK is expressed on many different epithelial tissues and epithelial tumour cells, and can activate specific downstream signalling pathways.

As actin polymerization is a hallmark of chemokine receptor signalling in cancer cell lines ${ }^{6,13}$, we speculated that RANKL and RANK might have a role in epithelial cell migration. In vitro stimulation of three different human breast cancer cell lines (MDA-MB-231, MCF-7 and Hs578T) with RANKL resulted in concentration-dependent cell migration, which was blocked using the decoy receptor OPG (Fig. 1d). Furthermore, RANKL triggered migration of two RANK-expressing prostate cancer cell lines, but had a negligible effect on migration of the colon cancer cell line Colo205, in which we failed to detect RANK expression (Fig. 1e). The extent of RANKL-induced migration in the breast cancer cell line MDA-MB-231 was comparable to the previously reported migration of these cells in response to the chemokines 6Ckine and CTAK ${ }^{6}$, but less than that observed with stromal cell-derived factor $1 \alpha$ (SDF-1 $\alpha$ ) (Fig. 1f and Supplementary Fig. 3). Although OPG inhibited RANKL-induced migration, it had no apparent effect on chemokineinduced migration (Fig. 1f). Thus, RANKL induces migration of malignant epithelial cells expressing RANK.

As physiological and malignant cell invasion use similar molecular mechanisms ${ }^{14}$, we evaluated the effects of RANKL on the migration of primary, non-transformed cells that express RANK. RANKLinduced cell migration was observed in primary mouse mammary epithelial cells freshly isolated from virgin females, and the primary, non-transformed mammary epithelial cell line MCF10A (Fig. 2a). Moreover, RANKL triggered directional migration of mature osteoclasts towards a RANKL source (see Supplementary Information and Supplementary Fig. 4). These data identify a role for the TNF/TNFR family molecules RANKL and RANK in the migration of primary breast epithelial cells and epithelial tumour cells.

${ }^{1}$ IMBA, Institute of Molecular Biotechnology of the Austrian Academy of Sciences, Dr. Bohr Gasse 3, A-1030 Vienna, Austria. ${ }^{2}$ Department of Medical Biophysics and ${ }^{3}$ Department of Immunology, University of Toronto, 610 University Avenue, Toronto, Ontario M5G 2C1, Canada. ${ }^{4}$ Ontario Cancer Institute, University Health Network, 610 University Avenue, Toronto, Ontario M5G 2C1, Canada. ${ }^{5}$ Faculty of Dentistry, McGill University, Montreal, Quebec H3A 1 A4, Canada. ${ }^{6}$ Amgen Inc., Thousand Oaks, California 91320-1799, USA. ${ }^{7}$ Division of Molecular and Life Sciences, Pohang University of Science and Technology, Pohang, 790-784 Kyungbuk, South Korea. ${ }^{8}$ Medical University of Vienna, Department of Obstetrics and Gynecology, Waehringer Guertel 18-20, A-1090 Vienna, Austria. ${ }^{9} \mathrm{CIHR}$ Group in Skeletal Development and Remodeling, Department of Physiology and Pharmacology and Division of Oral Biology, Schulich School of Medicine \& Dentistry, The University of Western Ontario, London, Ontario N6A 5C1, Canada. $\uparrow$ Present address: Faculty of Health Sciences, University of Ontario Institute of Technology, 2000 Simcoe Street North, Oshawa, Ontario L1H 7K4, Canada. *These authors contributed equally to this work. 
In contrast to most metastatic breast cancers ${ }^{2}$, some tumours, including malignant melanomas, metastasize to bone without stimulating osteoclastic resorption ${ }^{9}$. We found that a mouse B16F10 melanoma subclone ${ }^{15}$ expresses high levels of RANK mRNA (Supplementary Fig. 5a) and protein, as determined by RANKL-FITC binding (Fig. 2b). Similar to our results in breast and prostate cancer cells, RANKL had no apparent effects on proliferation or cell death in response to anisomycin, sorbitol or irradiation with ultraviolet light (not shown). Stimulation of B16F10 cells with RANKL caused actin polymerization (Fig. 2c) and increased cell migration (Fig. 2d). RANKL-induced migration of B16F10 melanoma cells was dependent on the concentration of
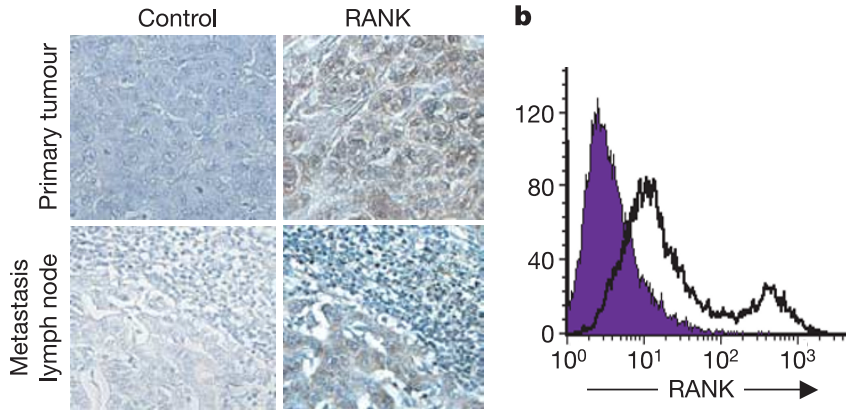

c
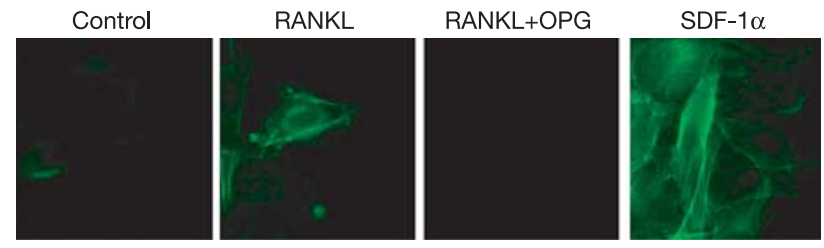

d
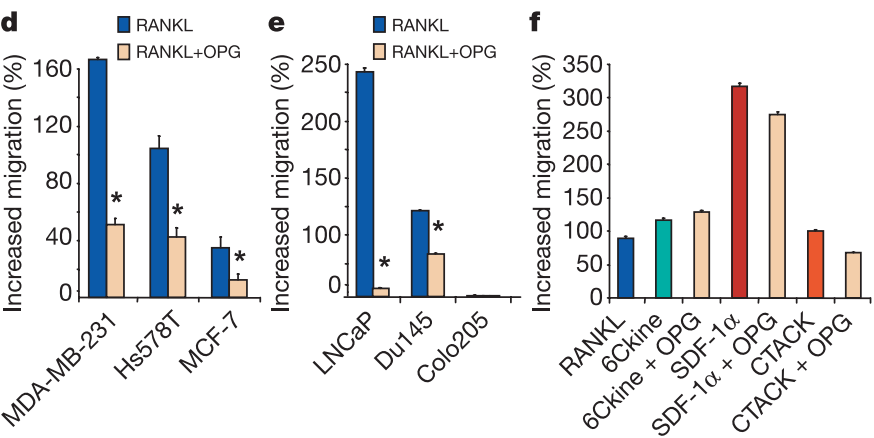

Figure 1 | RANK is expressed on breast cancer cells in patients and mediates migration of epithelial tumour cells. a, Expression of RANK on breast cancer cells at the site of the primary tumour and in lymph node metastases. Human breast and lymph node tissue arrays were stained with anti-RANK or control antibodies. Representative data are shown. Original magnification $\times 20$. b, Expression of RANK on MDA-MB-231 breast cancer cells. Background staining is shown in purple. c, rRANKL $\left(2.5 \mu \mathrm{g} \mathrm{ml}^{-1}\right)$ and SDF-1 $\alpha\left(80 \mathrm{ng} \mathrm{ml}^{-1}\right)$ trigger actin polymerization (detected by phalloidinFITC) in MDA-MB-231 cells. OPG $\left(10 \mu \mathrm{g} \mathrm{ml}^{-1}\right)$ blocks RANKL-induced actin polymerization. d, Migration of MDA-MB-231, Hs578T and MCF-7 human breast cancer cells in response to $\operatorname{rRANKL}\left(2.5 \mu \mathrm{g} \mathrm{ml}^{-1}\right) ; n=10$ experiments. Asterisk, OPG $\left(10 \mu \mathrm{g} \mathrm{ml}^{-1}\right)$ significantly reduced migration in MDA-MB-231 $(P<0.001)$, Hs578T $(P<0.0001)$ and MCF-7 $(P<0.005)$ cells. e, Migration of LNCaP and Dul45 human prostate carcinoma cells and Colo205 human colon cancer cells in response to $\operatorname{rRANKL}\left(2.5 \mu \mathrm{g} \mathrm{ml}^{-1}\right)$; $n=3$ experiments. Asterisk, OPG $\left(10 \mu \mathrm{g} \mathrm{ml}^{-1}\right)$ significantly reduced migration of LNCaP $(P<0.02)$ and Du145 $(P<0.05)$ cells. $f$, Migration of MDA-MB-231 cells in response to $\operatorname{rRANKL}\left(2.5 \mu \mathrm{g} \mathrm{ml}^{-1}\right)$ and the chemokines 6Ckine $\left(120 \mathrm{ng} \mathrm{ml}^{-1}\right), \operatorname{SDF}-1 \alpha\left(80 \mathrm{ng} \mathrm{ml}^{-1}\right)$ and CTACK $\left(100 \mathrm{ng} \mathrm{ml}^{-1}\right)$. There was no significant effect of $\mathrm{rOPG}\left(10 \mu \mathrm{g} \mathrm{ml}^{-1}\right)$ on chemokine-induced cell migration ( $n=3$ experiments). In $\mathbf{d}-\mathbf{f}$, the percentage increased migration ( \pm s.d.) compared to unstimulated control cells is shown.
RANKL and could be inhibited by the decoy receptor OPG (Fig. 2d). Stimulation of B16F10 cells with colony-stimulating factor-1 (CSF-1), which is required for RANKL-mediated osteoclastogenesis ${ }^{8}$, had no apparent effect on the migration of melanoma cells (Fig. 2d). The extent of RANKL-induced migration in B16F10 melanoma cells was comparable to the migration of these cells in response to the chemokines 6Ckine, CTAK and SDF-1 $\alpha$ (Supplementary Fig. 5b). Moreover, the effects of RANKL were additive with 6Ckine and CTACK, but not with SDF-1 $\alpha$ (Supplementary Fig. 5b), suggesting potential synergies between RANK and chemokine signalling that warrant further investigation. Treatment with OPG inhibited RANKL-, but not SDF-1 $1 \alpha$-induced migration,
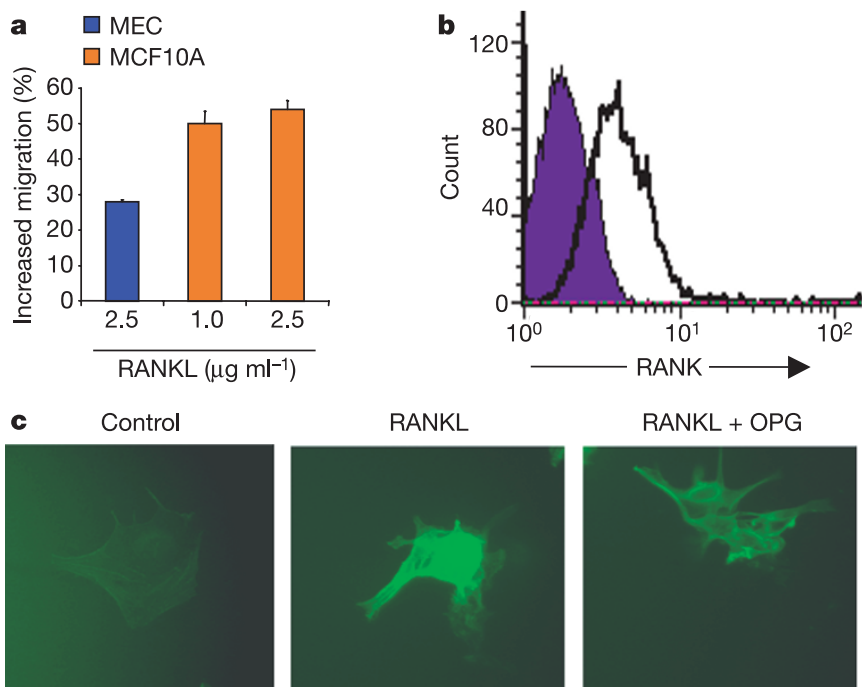

d
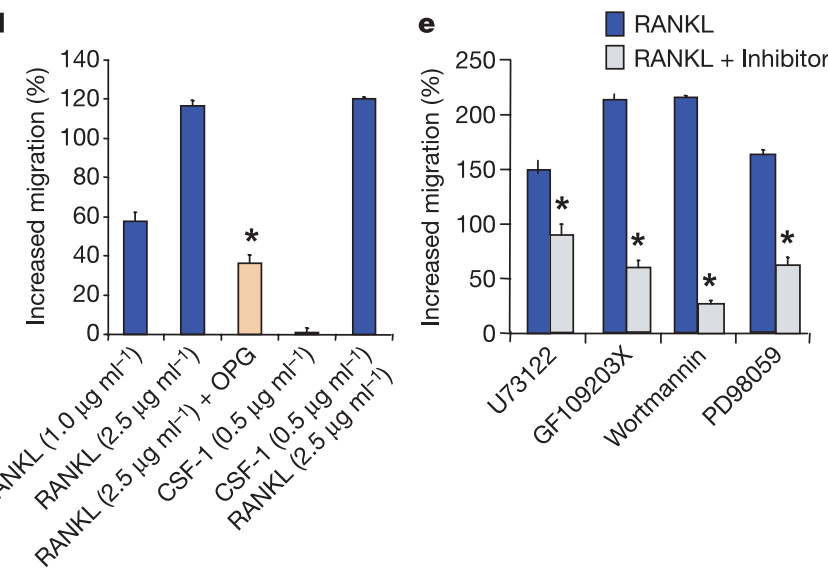

Figure 2 RANKL triggers migration of normal mammary epithelial cells and murine B16F10 melanoma cells. a, Migration of freshly isolated mouse mammary gland epithelial cells (MEC) and non-transformed human MCF10A breast epithelial cells in response to rRANKL. Percentage increased migration ( \pm s.d.) compared to non-stimulated control cells is shown. b, Surface expression of RANK on mouse B16F10 melanoma cells. Background staining is shown in purple. c, rRANKL $\left(2.5 \mu \mathrm{g} \mathrm{ml}^{-1}\right)$ triggers actin polymerization (phalloidin-FITC) in B16F10 cells that is blocked by rOPG $\left(10 \mu \mathrm{g} \mathrm{ml}^{-1}\right)$. d, Migration ( \pm s.d.) of B16F10 cells in response to rRANKL in the absence or presence of $\operatorname{rOPG}\left(10 \mu \mathrm{g} \mathrm{ml}^{-1}\right)$. Migration in response to CSF-1 is shown as a control. One result representative of ten experiments using different stimulation conditions is shown. Asterisk, $P<0.0004$ between samples treated with rRANKL and rRANKL + OPG. e, Migration ( \pm s.d.) of B16F10 cells in response to RANKL $\left(2.5 \mu \mathrm{g} \mathrm{ml}^{-1}\right)$ in the presence or absence of the inhibitors U73122 (10 $\mu \mathrm{M}$, PLC blocker), GF109203X (10 $\mu \mathrm{M}, \mathrm{PKC}$ blocker), wortmannin (100 nM, PI(3)K blocker) or PD98059 $(10 \mu \mathrm{M}, \mathrm{MEK} 1 / 2$ blocker). Asterisk, $P<0.05$ between samples treated with rRANKL and rRANKL + inhibitor. 
whereas inhibition of the SDF- $1 \alpha$ receptor with anti-CXCR4 antibody blocked SDF-1 $\alpha$-, but not RANKL-induced cell migration (Supplementary Fig. 5c). Phospholipase C (PLC), protein kinase C (PKC), ERK and phosphatidylinositol-3-OH kinase (PI(3)K) pathways have all been shown to be essential for chemokine-receptor induced cell migration, and we have recently shown that RANKL signals through PLC in osteoclasts ${ }^{16}$. Both RANKL and SDF- $1 \alpha$ stimulation of B16F10 melanoma cells induced actin polymerization (Fig. 2c) and ERK1/2 phosphorylation (Supplementary Fig. 5d), indicating that RANK and chemokine receptors use similar downstream signalling pathways. Inhibition of these signalling pathways inhibited RANKL-induced migration of this B16F10 melanoma subclone (Fig. 2e). These results indicate that in addition to chemokines, RANKL regulates migration of the mouse melanoma cell line B16F10.

To determine whether RANKL/RANK-regulated migration of cancer cells has a role in tumour metastasis in vivo, we analysed whether inhibition of RANKL/RANK through the decoy receptor OPG altered the metastasis into the bones. Intracardiac injection of mouse B16F10 melanoma cells into the left cardiac ventricle has previously been established as an in vivo model system to study metastasis into several organs, including the adrenal glands, the choroid plexus of the brain, the ovaries and bone 9 . Notably, the B16F10 subclone used in our experiments does not trigger osteoclast activation, a feature that allowed us to uncouple the direct effects of
RANKL on tumour metastasis from osteoclast-mediated effects ${ }^{17}$ (Supplementary Fig. 6a-c). Moreover, the tumour burden of B16F10 cells in vertebrae correlates with spinal invasion and paralysis as a functional disease read-out of bone metastasis (Fig. 3i and Supplementary Fig. 7a-c).

Injection of B16F10 melanoma cells into syngeneic C57BL/6 mice resulted in rapid metastasis of melanin-producing cancer cells into all long bones (Fig. 3a-c), vertebrae (Supplementary Fig. 7b), ovaries (Supplementary Fig. 7e), adrenal glands (Supplementary Fig. 7h) and the choroid plexus of the brain (not shown). Metastases were observed macroscopically in all animals analysed at days 12, 14 and 17 after injection, and virtually all bones in B16F10-injected mice showed black colour owing to the melanin-producing tumour cells (Fig. 3g). In vivo inhibition of RANKL with the decoy receptor OPG markedly reduced the melanin-producing B16F10 cancer foci and tumour burden in all bones at all time points analysed (Fig. 3d-h). In contrast, the tumour burden and metastasis of B16F10 melanoma cells into ovaries (Supplementary Fig. 7d-f), adrenal glands (Supplementary Fig. $7 \mathrm{~g}-\mathrm{i}$ ) and the brain (not shown) were comparable between control and OPG-treated animals. The progressive tumour growth in control vertebrae resulted in spinal cord invasion (Supplementary Fig. 7b) followed by clinical paralysis (Fig. 3i). However, treatment with OPG reduced the tumour burden in vertebrae (Supplementary Fig. 7c), and none of the OPG-treated mice developed clinical paralysis (Fig. 3i). Moreover, control mice injected with
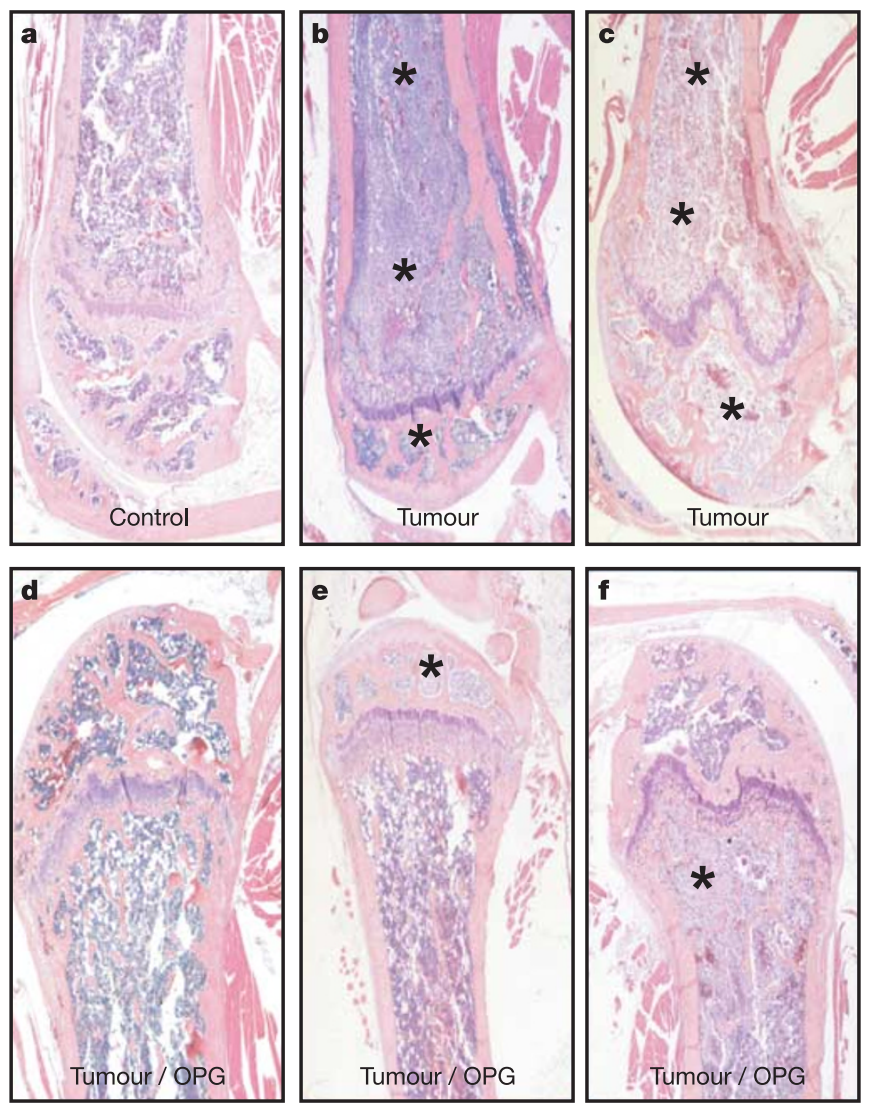

Figure 3 | Inhibition of RANKL/RANK signalling results in reduced tumour metastasis in the bones and abolishes paralysis. a-f, Histology of control long bones (a) and long bone on day 14 (b) or day 17 (c) after injection of B16F10 melanoma cells, and long bones on day $14(\mathbf{d}, \mathbf{e})$ or day 17 (f) after injection of B16F10 melanoma cells into mice treated with rOPG. Asterisks show typical examples of bone metastases for each treatment. Note that the tumour burden is markedly reduced in OPG-treated mice: no tumour metastasis into long bones (d), tumour foci in the metaphysis but not in other regions of the long bone (e), tumour foci in epiphysis and metaphysis but not in mid-diaphysis (f). Original magnification $\times 5$ for $\mathbf{a}-\mathbf{f}$.

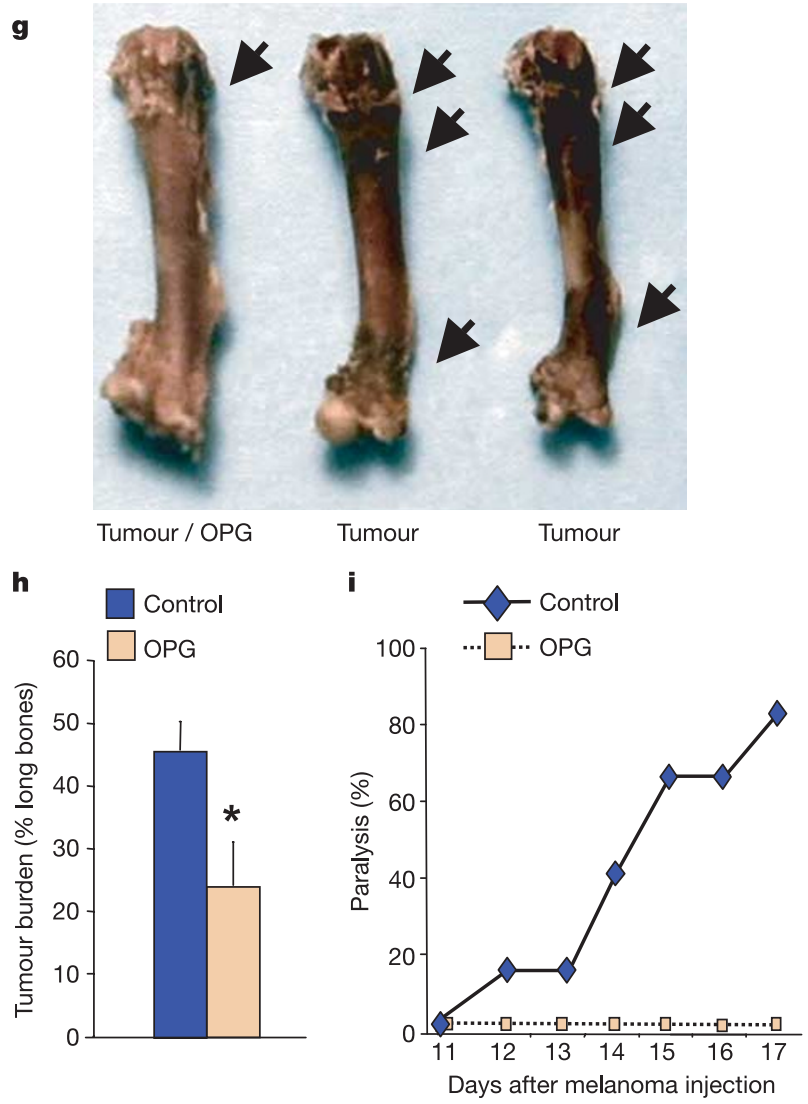

g, Macroscopic appearance of long bones on day 14 after injection of B16F10 cells into female recipients. Arrows indicate metastatic foci. Similar results (reduction in the tumour burden of OPG-treated mice) were observed in vertebrae, ribs and skull. $\mathbf{h}$, Tumour burden in long bones on day 14 after injection of B16F10 cells ( $n=12$ mice per group). Asterisk, $P<0.01$ between OPG-treated and untreated groups. Note that all long bones in the control animals showed metastases. The $y$-axis refers to the average burden ( \pm s.d.) of tumour cells in all long bones analysed. $\mathbf{i}$, Percentage of mice that developed hind leg paralysis on the indicated days following metastasis of $\mathrm{B} 16 \mathrm{~F} 10$ cells into vertebrae ( $n=12$ per group). 
B16F10 cells showed high morbidity, with some mice dying before the end of the experiment, whereas none of the OPG-treated animals died within the experimental time frame (not shown). Treatment of mice with the bisphosphonate zoledronic acid ${ }^{18}$ did not change the tumour burden of B16F10 cancer metastases in bones. Furthermore, using the same experimental system and immunodeficient mice as hosts, the human colon cancer cell lines SW480 and Colo205, which do not express detectable levels of RANK, failed to metastasize into the bones after intracardiac injection $(n=45$ mice). Thus, in vivo inhibition of RANKL with OPG can selectively abrogate metastasis and the tumour burden of B16F10 melanoma cells in bones.

The organ preference of metastatic colonization is influenced by communication between the circulating tumour cells and the target host tissue ${ }^{1-4,14}$. In particular, osteotropism of certain malignancies is a complication of the primary cancer that often results in severe bone destruction, hypercalcaemia and intractable skeletal pain ${ }^{5}$. In fact, metastases, rather than primary tumours, are responsible for most cancer deaths ${ }^{1}$, and it has been estimated that $70 \%$ of patients with progressive breast cancer and $84 \%$ of prostate cancer patients develop bone metastases ${ }^{1,2,19}$.

It has long been unclear as to why particular cancers preferentially metastasize to bones. The environment of resorbing bone can provide nutrients to cancer cells, and tumour cells can express osteoclastogenic factors such as parathyroid hormone-related protein (PTHrP) that contribute to local bone degradation and cancer growth $^{2,19-21}$. Organ-specific chemoattractant molecules have recently been implicated in the preferential homing of breast cancer cell lines to tissues such as lung and lymph nodes ${ }^{5,6}$. For instance, the chemokine receptor CXCR4 is highly expressed in malignant breast cancer cells, and its ligand, SDF- $1 \alpha$, is found in organs to which breast cancer frequently metastasizes ${ }^{6}$. However, as inhibition of chemokine receptors in vivo only partially blocks the metastatic behaviour of breast cancer cells ${ }^{6}$, other factors must exist to control the tissue-specific migration of epithelial cancer cells.

RANKL is a critical osteoclast differentiation factor that is highly expressed in the bone marrow environment ${ }^{8}$. As we found expression of the receptor RANK on cells from multiple epithelial tumours and a malignant melanoma cell line, which preferentially metastasize to bone, we speculated that RANKL might be one of the long soughtafter 'soil' factors ${ }^{4}$ that facilitates metastasis to bone. Our results show that RANKL triggers cytoskeletal changes and migration of several human epithelial tumour cells that express RANK. RANKL also stimulates migration of primary breast epithelial cells and osteoclasts, establishing that RANKL-induced cell migration also occurs in normal, non-transformed cells. Importantly, inhibition of RANKL/RANK signalling by OPG in vivo markedly and selectively reduces bone metastasis and tumour burden in a melanoma model that does not activate osteoclasts ${ }^{15}$. It remains to be determined whether the dynamics of membrane-bound RANKL and its cleavage to the soluble form present in the plasma of humans and mice could contribute to the metastasis of melanoma cells ${ }^{8,11}$ or metastasis in other model systems ${ }^{22}$.

In conclusion, our data establish that RANKL can act as a tissuespecific factor for migration of cancer cells and that RANKL is a prominent 'soil' factor for bone-specific metastases of epithelial tumours. Therefore, inhibition of RANKL-RANK interactions may offer a promising therapeutic target for interfering with tumour metastasis and progression in bones.

\section{METHODS}

Tumour cell lines. B16F10 murine melanoma cells, MDA-MB-231 human breast cancer, MCF-7 human breast cancer, Hs578T human breast cancer, Colo205 human colon cancer, SW480 human colon cancer, LNCaP human prostate cancer, Du145 human prostate cancer and T47D human epithelial breast tumour cells were used. Non-transformed MCF10A mammary gland epithelial cells and primary mouse mammary gland epithelial cells were freshly isolated from non-pregnant C57BL/6 mouse mammary glands. Animal experiments were performed in accordance with the guidelines of the Council on Animal Care at the University of Toronto and the University of Western Ontario.

RANKL and RANK expression analysis. Total RNA was isolated from cell lines and mouse tissues using Trizol (Invitrogen), and RANK (Tnfrsf11a gene) and RANKL (Tnfsf11 gene) mRNA expression were analysed by polymerase chain reaction (PCR). In some experiments, RANK transcripts were confirmed by quantitative real-time PCR with reverse transcription (RT-PCR). RANK mRNA levels were normalized to $\beta$-actin levels. Detection of cell-surface expression of RANK protein by fluorescence-activated cell sorting (FACS) used FITC-conjugated human RANKL (amino acids 159-317; Amgen).

RANK signalling, proliferation and cell death assays. Cancer cells were serumstarved for $12 \mathrm{~h}$ and then stimulated with recombinant murine RANKL (amino acids 158-316) in the presence or absence of recombinant murine OPG-FC protein (amino acids 22-401; rOPG, both from Amgen) ${ }^{11}$, SDF-1 $\alpha$ (R\&D Systems) or recombinant prolactin (Sigma). In addition, commercially available RANKL (R\&D Systems) was used with similar results in osteoclastogenesis, indicating that the observed effects were not attributable to secondary effects of recombinant RANKL (amino acids 158-316).

For western blotting, antibodies reactive to ERK1/ERK2, active ERK1/ERK2 (phosphorylated on Thr 202 and Tyr 204), PKB/AKT, active PKB/AKT (phosphorylated on Ser 473), STAT5A/B, phospho-Stat5A/B (phosphorylated on Tyr 694) (Cell Signalling and Transduction Lab) and actin (Sigma) were used. For actin polymerization studies, tumour cells were stimulated with RANKL or SDF- $1 \alpha$, and actin polymerization was determined using phalloidin-FITC. Tumour cell proliferation was determined using ${ }^{3} \mathrm{H}$-thymidine uptake. Cell death was detected by FACS using propidium iodide/AnnexinV-FITC double staining.

Tumour cell migration. Migration of cancer cells was assessed using a 96-well chemotaxis chamber (NeuroProbe Inc.) with fibronectin- (Sigma) coated polycarbonate filters ( 8 - and $12-\mu \mathrm{m}$ pore size). All cells were starved for $12 \mathrm{~h}$ in DMEM (10 mM HEPES, $0.1 \%$ bovine serum albumin), detached using $5 \mathrm{mM}$ EDTA in $\mathrm{Ca}^{2+} / \mathrm{Mg}^{2+}$-free Hank's buffer, counted and resuspended for each assay. rRANKL, rOPG or the chemokines SDF-1 $\alpha$, 6Ckine and CTACK (all chemokines were purchased from $\mathrm{R} \& \mathrm{D}$ Systems) were placed in the lower wells and $5 \times 10^{5} \mathrm{~B} 16 \mathrm{~F} 10$ cells or $2 \times 10^{5}$ human breast, prostate or colon cancer cells were placed in the upper wells. Migration of cells was determined at $37^{\circ} \mathrm{C}$ for $16 \mathrm{~h}$ (B16F10 cells) or $6 \mathrm{~h}$ (human cancer cells) as previously described ${ }^{6}$.

RANK detection on human breast cancer tissue arrays. Paraffin-embedded specimens of tumours, lymph node metastasis, and adjacent normal tissue were collected from 59 female breast cancer patients who underwent surgery between 1988 and 1994, and were analysed retrospectively using protocols approved by the institutional review board of the Medical University of Vienna. Triplicate core biopsies of $0.6 \mathrm{~mm}$ were taken from each donor paraffin block and arrayed. Paraffin sections (5- $\mu \mathrm{m}$ thick) were treated in xylene and rehydrated in a gradient of ethanol. After antigen retrieval by $10 \mathrm{mM}$ sodium citrate (pH 6.0), sections were incubated with a goat polyclonal anti-RANK antibody (M-20, Santa Cruz) for $1 \mathrm{~h}$. Sections were then incubated with biotinylated anti-goat/ rabbit IgG antibodies, followed by incubation with streptavidin-peroxidase and 3,3'-diaminobenzidine. Immunostaining was scored on triplicate tissues by two independent observers (T.N. and R.S.) using the following arbitrary scale: 0 , no staining; 1, weak staining; 2, medium staining; 3 , strong staining. It should be noted that all cancer tissues showed staining in more than $50 \%$ of the total tumour area.

In vivo tumour metastasis. Murine B16F10 melanoma cells or human colon cancer cells that do not express RANK were injected into the left cardiac ventricle of 7-10-week-old female C57BL/6 mice or nude mice, respectively ${ }^{9}$. Simultaneously, mice were daily treated with vehicle (PBS), $20 \mu \mathrm{g}$ rOPG per day, or zolendronic acid ( $3 \mu \mathrm{g}$ per mouse per day, subcutaneously) as previously described ${ }^{18}$. After the final treatment, mice were killed, and bones (femur, tibia, humerus and lumbar vertebrae) and organs (brain, ovary, spleen, kidney and adrenal glands) were collected for histological analysis. Radiographic and histomorphometric analysis of all bones was as previously described ${ }^{7,21}$. Briefly, tissues were fixed in $10 \%$ formalin, sectioned and stained with haematoxylin and eosin to determine the presence of metastases. Midline longitudinal sections of long bones were stained for tartrate-resistant acid phosphatase activity. Two non-serial sections of each bone were assessed. The total tissue section area and the tissue area occupied by tumour cells were measured using the Osteomeasure bone analysis program (Osteometrics Inc.).

\section{Received 11 November; accepted 12 December 2005}

1. Chambers, A. F., Groom, A. C. \& MacDonald, I. C. Dissemination and growth of cancer cells in metastatic sites. Nature Rev. Cancer 2, 563-572 (2002). 
2. Mundy, G. R. Metastasis to bone: causes, consequences and therapeutic opportunities. Nature Rev. Cancer 2, 584-593 (2002).

3. Sloan, E. K. \& Anderson, R. L. Genes involved in breast cancer metastasis to bone. Cell. Mol. Life Sci. 59, 1491-1502 (2002).

4. Paget, $\mathrm{S}$. The distribution of secondary growths in cancer of the breast. Lancet 1, 571-572 (1889).

5. Moore, M. A. The role of chemoattraction in cancer metastases. Bioessays 23, 674-676 (2001)

6. Muller, A. et al. Involvement of chemokine receptors in breast cancer metastasis. Nature 410, 50-56 (2001).

7. Kong, Y. Y. et al. OPGL is a key regulator of osteoclastogenesis, lymphocyte development and lymph-node organogenesis. Nature 397, 315-323 (1999).

8. Lacey, D. L. et al. Osteoprotegerin ligand is a cytokine that regulates osteoclast differentiation and activation. Cell 93, 165-176 (1998).

9. Arguello, F., Baggs, R. B. \& Frantz, C. N. A murine model of experimental metastasis to bone and bone marrow. Cancer Res. 48, 6876-6881 (1988)

10. Anderson, D. M. et al. A homologue of the TNF receptor and its ligand enhance T-cell growth and dendritic-cell function. Nature 390, 175-179 (1997)

11. Teitelbaum, S. L. Bone resorption by osteoclasts. Science 289, 1504-1508 (2000).

12. Fata, J. E. et al. The osteoclast differentiation factor osteoprotegerin-ligand is essential for mammary gland development. Cell 103, 41-50 (2000).

13. Verschueren, $\mathrm{H}$. et al. Metastatic competence of BW5147 T-lymphoma cell lines is correlated with in vitro invasiveness, motility and $\mathrm{F}$-actin content. J. Leukoc. Biol. 55, 552-556 (1994).

14. Liotta, L. A. \& Kohn, E. C. The microenvironment of the tumour-host interface Nature 411, 375-379 (2001).

15. Bakewell, S. J. et al. Platelet and osteoclast $\beta_{3}$ integrins are critical for bone metastasis. Proc. Natl Acad. Sci. USA 100, 14205-14210 (2003).

16. Komarova, S. V., Pilkington, M. F., Weidema, A. F., Dixon, S. J. \& Sims, S. M. RANK ligand-induced elevation of cytosolic $\mathrm{Ca}^{2+}$ accelerates nuclear translocation of nuclear factor kB in osteoclasts. J. Biol. Chem. 278, 8286-8293 (2003).

17. Sanchez-Sweatman, O. H., Lee, J., Orr, F. W. \& Singh, G. Direct osteolysis induced by metastatic murine melanoma cells: role of matrix metalloproteinases. Eur. J. Cancer 33, 918-925 (1997).

18. Peyruchaud, O. et al. Early detection of bone metastases in a murine model of fluorescent human breast cancer cells: application to the use of the bisphosphonate zoledronic acid in the treatment of osteolytic lesions. J. Bone Miner. Res. 16, 2027-2034 (2001).

19. Guise, T. A. Molecular mechanisms of osteolytic bone metastases. Cancer 88 (Suppl.), 2892-2898 (2000).

20. Zhang, J. et al. Osteoprotegerin inhibits prostate cancer-induced osteoclastogenesis and prevents prostate tumour growth in the bone. J. Clin Invest. 107, 1235-1244 (2001)

21. Morony, S. et al. Osteoprotegerin inhibits osteolysis and decreases skeletal tumour burden in syngeneic and nude mouse models of experimental bone metastasis. Cancer Res. 61, 4432-4436 (2001)

22. Lelekakis, M. et al. A novel orthotopic model of breast cancer metastasis to bone. Clin. Exp. Metastasis 17, 163-170 (1999).

Supplementary Information is linked to the online version of the paper at www.nature.com/nature.

Acknowledgements These studies were supported in part by grants from the Canadian Institutes of Health Research (CIHR/IMHA/TAS) and the Canadian Arthritis Network to S.M.S., S.J.D. and S.V.K. We thank W. Boyle, D. Lacey and C. Dunstan for providing rRANKL, RANKL-FITC and rOPG. J.M.P. is supported by the National Cancer Institute of Canada, IMBA, the Austrian National Bank and a European Union Marie Curie Excellence Grant. T.N. holds a European Union Marie Curie Mobility Fellowship.

Author Information Reprints and permissions information is available at npg.nature.com/reprintsandpermissions. The authors declare competing financial interests: details accompany the paper on www.nature.com/nature. Correspondence and requests for materials should be addressed to J.M.P. (josef.penninger@imba.oeaw.ac.at). 